\title{
BRAIN AGING AND PARKINSON'S DISEASE: NEW THERAPEUTIC APPROACHES USING DRUG DELIVERY SYSTEMS
}

\author{
C. Rodríguez-Nogales ${ }^{1}$, E. Garbayo ${ }^{1,2}$, M.M. Carmona-Abellán ${ }^{3}$, M.R. \\ Luquin $^{2,3}$, M.J. Blanco-Prieto ${ }^{1,2 *}$ \\ ${ }^{1}$ Pharmacy and Pharmaceutical Technology Department, University of Navarra, Spain \\ ${ }^{2}$ Instituto de Investigación Sanitaria de Navarra (IDISNA), Pamplona, Spain \\ ${ }^{3}$ Laboratory of Regenerative Therapy, Department of Neurology and Neuroscience \\ Division, Centre for Applied Medical Research (CIMA), University of Navarra, Spain
}

*Correspondence to: M.J. Blanco-Prieto, Department of Pharmacy and Pharmaceutical Technology, School of Pharmacy, University of Navarra, C/Irunlarrea 1, 31080 Pamplona, Spain. Tel: +34 948425600 x 6519, Fax: 34 948425649. E-mail address: mjblanco@unav.es 


\begin{abstract}
The etiology and pathogenesis of Parkinson's disease (PD) is unknown, aging being the strongest risk factor for brain degeneration. Understanding PD pathogenesis and how aging increases the risk of disease would aid the development of therapies able to slow or prevent the progression of this neurodegenerative disorder. In this review we provide an overview of the most promising therapeutic targets and strategies to delay the loss of dopaminergic neurons observed both in PD and aging. Among them, handling alphasynuclein toxicity, enhancing proteasome and lysosome clearance, ameliorating mitochondrial disruptions and modifying the glial environment are so far the most promising candidates. These new and conventional drugs may present problems related to their labile nature and to the difficulties in reaching the brain. Thus, we highlight the latest types of drug delivery system (DDS)-based strategies for PD treatment, including DDS for local and systemic drug delivery. Finally, the ongoing challenges for the discovery of new targets and the opportunities for DDS-based therapies to improve and efficacious PD therapy will be discussed.
\end{abstract}

\title{
Keywords
}

Parkinson disease, aging, therapeutic targets, alpha-synuclein, GDNF, drug delivery systems, micro/nanoparticles. 


\begin{abstract}
Abbreviations
PD, Parkinson's disease; DAergic, dopaminergic; SNpc, substantia nigra pars compacta; DA, dopamine; LB, lewy body; $\alpha$-syn, alpha synuclein; L-DOPA, levodopa; MAO, monoamine oxidase; CNS, central nervous system; DDS, drug delivery systems; MPs, microparticles; NPs, nanoparticles; SNCA, synuclein alpha non A4 component of amyloid precursor; PLK, Polo like kinase; LAMP, lysosomal associated membrane receptor protein; LRRK2, leucine-rich repeat kinase 2; MPTP, 1-methyl-4-phenyl1,2,3,6-tetrahydropyridine; GDNF, glial cell line-derived neurotrophic factor; BBB, blood-brain barrier; VEGF, vascular endothelial growth factor; PEG, polyethylen glycol; PLGA, poly(lactic-coglycolic acid).
\end{abstract}




\section{INTRODUCTION}

Parkinson`s disease (PD) is a neurodegenerative disorder characterized by the progressive loss of dopaminergic (DAergic) neurons of the substantia nigra pars compacta ( $\mathrm{SNpc}$ ), especially the DAergic neurons of the ventrolateral tier that project to the striatum [1]. The differential vulnerability of DAergic neurons to degeneration is still a matter of debate, but it seems to be related to increased sensitivity to mitochondrial dysfunction and changes in protein degradation [2-4]. The consequent decrease in striatal dopamine (DA) is correlated with motor impairment symptoms such as tremor, bradikynesia and rigidity. Non-motor symptoms such as sleep disorders or depression are also present during the disease, just as neuronal loss may occur in other brain regions. Lewy bodies (LBs) and neurites are intracellular inclusions that constitute the neuropathological hallmark of PD due to an abnormal protein aggregation of alphasynuclein ( $\alpha$-syn) predominantly [5].

The etiology of PD is unknown although researchers believe that it may be caused by a combination of environmental and genetic factors, aging being the strongest risk factor $[6,7]$. The rate of progression of PD yields evidence of the natural loss of compensatory mechanisms associated with aging. In this way early-onset PD leads to a slower deterioration over time than late-onset PD [8]. The link or difference between aging and PD may be the intensity or acceleration of the neurological damage processes [9]. This imbalance of compensatory resources has led specialists to seek novel knowledge about the aging process.

At present there is no cure for PD and current therapies are focused on DA replacement. Levodopa (L-DOPA) still remains the most effective drug for controlling PD symptoms $[10,11]$. However, as the disease progresses, many PD patients become severely disabled due to the appearance of non-DAergic symptoms, particularly in the advanced stage of the disease and to the occurrence of drug-induced side effects such as fluctuations [12]. In order to produce a marked benefit not only on motor symptoms but also on non-motor symptoms, non ergotic DA agonists such as pramipexol, ropinirol and rotigotine are now used [13]. Other adjuvant drugs block enzymes that break down L-DOPA/DA such as catechol- $O$-methyltransferase inhibitors and monoamine oxidase B (MAO-B) inhibitors inducing a longer duration of L-DOPA effect and decreasing fluctuations. Finally, the DA agonist apomorphine is used as a rescue therapy when unpredictable off periods appear along the day and the glutamate activity blocker amantadine is used as a potent antidyskinetic agent. Certain non-motor symptoms with a great impact on the quality of life do not respond to dopaminergic drugs and, consequently, new drugs that cover this gap are essential [14]. In addition, the development of a disease-modifying strategy has become a priority. In this regard, much has been learned in recent years about PD pathophysiology. Most of these discoveries have identified potential targets, which may lead to the development of novel PD therapies. Remarkably, most of the new drugs and potential therapies for PD are labile compounds that show short in vivo half-life and that come with the challenges of delivery to the central nervous system (CNS). Thus, innovative technologies able to 
extend the length of the treatment, release drugs in a controlled manner or enhance brain drug delivery are the key to developing more effective treatments. In this regard, drug delivery systems (DDS) such as liposomes, microparticles (MPs), nanoparticles (NPs) or hydrogels, as well as conventional transdermal patches or subcutaneous implants, have shown great potential for improving the outcome of new and conventional therapies for PD [15]. The aim of this paper is to review novel therapeutic targets that may detain DAergic neurodegeneration observed both in PD and aging. Then, recent advances in the use of DDS for PD are discussed. Finally, the ongoing challenges for the discovery of new targets and the opportunities for DDS-based therapies for an improved and efficacious PD therapy will be discussed.

\section{METHODS}

\section{Evaluation of the literature}

A MEDLINE search was conducted using the keywords aging, therapeutic targets, alpha-synuclein, mitochondrial disruptions, neuroinflammation, glia and Parkinson disease. For the section "Drug delivery systems approaches" the literature search, from 2013 to 2015, included the keywords drug delivery systems, microparticles, nanoparticles, GDNF and Parkinson disease. All research papers and reviews with the mentioned contents were included in the review process.

\section{NEW THERAPEUTIC TARGETS}

Recent knowledge of the basic mechanisms and pathophysiology of PD might support the search for targets for treating this disease. In this section we will review the most promising therapeutic targets and strategies to detain the loss of DAergic neurons (Figure 1).

\subsection{Alpha-synuclein toxicity. Phosphorylation and aggregation}

This small peptide of 140 amino acids is the main LB component and it seems to be closely linked to PD pathogenesis [5] (Figure 1A). However, the role of $\alpha$-syn on disease onset and progression is not totally understood. Physiologically, $\alpha$-syn regulates synaptic activity by interacting with synaptic vesicles and promotes a snare complex assembly [16]. Interestingly, $\alpha$-syn expression is increased with aging [8].

The implication of synuclein in PD results from the identification of mutations in the SNCA gene (synuclein alpha non A4 component of amyloid precursor) in PD families. Duplication or triplication of SNCA (PARK4 locus) has also been implicated in PD, indicating that $\alpha$-syn-wild type may trigger toxicity at high levels [7]. Point mutations in SNCA gene (PARK1 locus) affecting $\alpha$-syn conformation are also associated with familial PD, thus targeting $\alpha$-syn post translational modifications has also drawn the 
attention of various authors to date and researchers have consequently focused on targeting $\alpha$-syn for therapeutic intervention.

In PD an aggregation balance may exist between $\alpha$-syn oligomers, amyloid fibrils and LBs. This event might be a protective mechanism functioning like a garbage storing system, while the previous oligomer state might be the pathologic version. In this regard, phospho- $\alpha$-syn has been reported to enhance oligomer and LB formation [17]. Phosphorylation of $\alpha$-syn at serine 129 is characteristic of PD, polo-like kinase 2 (PLK2) being the enzyme responsible for $\alpha$-syn phosphorylation [18]. PLK2 has been reported to activate an antioxidant pathway to promote the survival of cells with mitochondrial dysfunction [19]. Furthermore, its gene is transcriptionally responsive to calcium and ROS (reactive oxygen species) signals. Additionally, PLK2 may phosphorylate PLK1 allowing bypass of DNA damage checkpoint cell cycle progression as well as participating in centrosome duplication [20]. On the grounds of a putative neurotoxic attribute, kinase activity modulators are being studied. Nevertheless, some controversial outcomes also support a neuroprotective role for PLK2. The difficulty in matching PLK2 implications could reside in the fact that this molecule is involved in several physiological pathways. Following this hypothesis, our group is at present working to validate this interesting target.

Various pieces of evidence demonstrate a cell-to-cell transmission of $\alpha$-syn as a prionlike propagation process [21]. Although more studies are needed to confirm this event, immunotherapy focused on the clearance of this aggregates is now considered to be a new disease-modifiying therapy [22]. Immunotherapy is turning into an increasingly tangible reality and may offer promising neuroprotective drugs that could manage $\alpha$-syn aggregation, spread or clearance. Recent studies in animal models of the disease have reported neuroprotection after passive or active immunization [4]. This approach may stop $\alpha$-syn transcellular propagation but not intraneuronal inclusions, making it necessary to optimize intrabodies in order to detain intracellular $\alpha$-syn as well [23].

\subsection{Proteasome and lysosome approach.}

The ubiquitin-proteasome system and the autophagic-lysosomal processes represent the two major degradation pathways for proteins including $\alpha$-syn (Figure 1B). Both systems show a reduction in function and efficiency with age. There is evidence that soluble and oligomeric forms of $\alpha$-syn are ubiquitinated and then degraded by the proteasome. Thus, impairment of ubiquitin proteasome system may lead to intracellular inclusions in PD [24]. Consequently, proteasome system activators should be investigated to design new drugs that facilitate $\alpha$-syn clearance. In this regard, defective proteins are tagged via ubiquitination for proteasome selective destruction and the E3 ubiquitin protein ligase (parkin) mutation has been linked to familial PD [24].

The accumulation of the aged pigment lipofuscin as a lysosomal degradation product of damaged mitochondria indicates that macro autophagy is a neuroprotective mechanism 
during aging [8]. Macro autophagy constitutes the unique mechanism to recycle entire organelles such as mitochondria [25]. Chaperone-mediated autophagy forms part of the third lysosome process in which a specific cytosolic protein-molecular chaperone (hsc70) binds to the lysosomal associated membrane receptor protein 2 (LAMP2A), before transporting inside the lysosome [26]. $\alpha$-syn is a known substrate of chaperone mediated autophagy and it has been recently reported that $\alpha$-syn overexpression is correlated with a decrease of LAMP-1, indicating an impairment of the phagocytic ability and generating a mitophagy dysfunction [27]. Dehay et al. proposed that glucosidase beta acid gene mutation that causes Gaucher's disease and lysosomal ATPase transporter ATP13A2/PARK9 mutation may be related to an $\alpha$-syn accumulation [28]. Murphy et al. have reported that LAMP2 protein isoforms are affected in different ways in PD [29] and Xilouri et al suggested that overexpressing LAMP2A, may decrease $\alpha$-syn-induced neurodegeneration [26]. More investigations are needed to develop new molecules that enhance the lysosome-autophagy system improving $\alpha$-syn degradation. Leucine-rich repeat kinase 2 (LRRK2) mutations represent a common genetic cause of PD and are implicated in various functions in which autophagy may be linked to PD [7]. Lee et al. demonstrated the neuroprotective potential of LRRK2 kinase inhibitors in LRRK2-induced neurodegeneration model [30].

\subsection{Mitochondrial disruptions and oxidative stress.}

Mitochondrial dysfunction has been involved in the etiopathogenesis of PD (Figure 1C). Thus, mitochondrial-targeted therapies directed to up-regulate mitochondrial function have emerged as potential therapeutic targets for PD. Although PD is a consequent interplay of several factors, 1-methyl-4-phenyl-1,2,3,6-tetrahydropyridine (MPTP) and other selective DA neurotoxins that inhibit mitochondrial complex currently used to create PD models support the mitochondrial impairment in PD [31]. PTEN-induced putative kinase 1 (PINK1) and E3 ubiquitin protein ligase (PARKIN) mutations involve different proteins and processes related to axonal transport, fusion/fission and mitophagy of damaged mitochondria [9] and have been implicated in familial PD. $\alpha$-syn is also suggested to interact with membranes and organelles including mitochondria. In this sense, Bir et al. report that $\alpha$-syn induces mitochondrial disruption in vitro [32]. Their study proposes that $\alpha$-syn binds mitochondria, opening membrane permeability transition pores and triggering a potential transmembrane breakdown. Besides, LRRK2 mutation, has recently been linked with mitochondrial degeneration [31].

Mitochondria are a well-known source of oxidative stress. Oxidative stress plays an important role both in PD and aging (Figure 1C) According to this, and importantly, the protein deglycase DJ-1, a protector against oxidative stressors, is defective in both PD and aging [9]. Besides the fact that ROS and free radicals like $\mathrm{H}_{2} \mathrm{O}_{2}$ and $\mathrm{OH}^{-}$, are generated in the mitochondria, some perspectives support the view that the accumulation of cytosolic DA metabolites can be correlated with oxidative and nitrative damage to dopaminergic cells [8]. The DA transporter and the vesicular monoamine 
transporter 2 may possess a defensive role in this regard, being in charge of withdrawal of the excess production of free radicals. In this regard, antioxidant replacement therapies play an important role in the search for novel drugs for neurodegenerative disorders of aging. On the other hand, $\mathrm{Ca}^{2+}$ dyshomeostasis has frequently been thought to be a consequence of cell damage in PD. The stress which arises between the endoplasmic reticule and the mitochondria to meet the requirement of sustained release of $\mathrm{Ca}^{2+}$ could contribute to LB formation and premature death of SNpc DA neurons [33]. Although this is not fully understood at present, use of approved $\mathrm{Ca}^{2+}$ channel antagonists may be assessed to attempt neuroprotection in PD.

\subsection{Glial environment and neuroinflammation}

Not only intracellular mechanisms but also the surrounding environment of the DAergic neurons have been described as contributing to disease progression and aging (Figure 1D). Microglia have been described to like macrophages, secreting neuroinflammatory cytokines and leading cell degeneration [9]. Astrocytes support neuroprotection, changing their morphology or overexpressing glial fibrillary acidic protein. However, astrocyte activation has also been linked to most of the pathologies of the brain, including PD. Targeting the neuroinflammatory process may be a suitable strategy to cease PD progression. Umemura et al. designed a longitudinal retrospective analysis associating C-reactive protein with motor deterioration in parkinsonian patients showing that the subclinical elevation of this marker in plasma is linked to motor prognosis in PD [34]. Brundin et al. suggested that immunosuppression may reduce neurodegeneration, given that $\alpha$-syn is an immune response inducer [22]. In this sense, calcineurin and tumor necrosis factor inhibitors were assayed achieving a cell survival enhancement and $\mathrm{T}$ cell infiltration decrease in vivo. Moreover, anti-inflammatory drugs like minocycline and non-steroidal anti-inflammatory agents are currently being investigated as potential neuroprotective therapies [3]. Finally, the glucagon-like peptide 1 receptor agonist exenatide, a drug used to treat type 2 diabetes, is currently being studied in clinical trials with parkinsonian patients [22].

The development of new drugs should be focused on activating proper neuroprotective and neurotrophic pathways as well as to trigger neurogenesis and gliogenesis. In this regard, it is useful to bear in mind the neurogenesis and gliogenesis provided by the subventricular zone as a compensatory mechanism and their possible involvement in PD and aging [9]. Neurotrophic factors such as glial cell-line derived neurotrophic factor (GDNF) could be of interest.

\section{DRUG DELIVERY SYSTEM APPROACHES}

To develop novel therapies from the strategies detailed above it is essential to devise suitable formulations to enhance and preserve their clinical potential. Many candidates and established drugs are labile molecules with short in vivo half-life that require a DDS to reach clinical applications. DDS are biodegradable or non-biodegradable formulations that offer improvements in the protection, absorption, penetration and 
distribution of drugs (Figure 2). Innovative delivery systems are able to avoid first pass effect, allow sustained drug release over time, cross the blood brain barrier (BBB) and reduce toxic effects. Our previous work reviewed a detailed list of the DDS studies performed between 2008 and 2013 in the context of PD [15]. Here, we review studies published since 2013.

\subsection{DDS for local delivery}

DDS used for local treatment can be injected by stereotaxic surgery in a minimally invasive way in discrete precise and functional brain areas without damaging the surrounding tissue. Polymeric MPs or NPs may allow sustained release of drug over months, avoiding not only multiple administrations but also the complications related to this kind of intervention.

\section{$\underline{D D S}$ for dopamine delivery}

In order to restore DA content, a novel DA DDS was proposed encapsulating non autologous DA-secreting cells on a hybrid scaffold of alginate gel/3D framework [35]. Pores in alginate gel allow the transfer of oxygen, nutrients or hormones and block host patient antibodies. Although in vitro studies demonstrate a suitable DA release over 8 weeks, appropriate in vivo studies are required to determine consistent clinical application.

\section{$\underline{D D S}$ for neurotrophic factor delivery}

One of the most exciting approaches for PD treatment is the use of the diseasemodifying growth factor GDNF. Our laboratory obtained interesting outcomes by administrating biodegradable GDNF-MPs in rat [36,37] and monkey model [38] of PD. In rats, GDNF-MPs achieved long-term neuroprotection and neurorestoration demonstrating that MP are an efficient vehicle for sustained neurotrophic factor delivery to the brain [36,37]. In addition, parkinsonian monkeys showed brain structural and functional recovery following local delivery of microencapsulated GDNF [38]. Herran et al. designed a combinatorial strategy by nanoencapsulating vascular endothelial growth factor (VEGF) and GDNF [39]. Motor function restoration and enhancement of DAergic neurons in the SN was observed after VEGF- and GDNF-loaded NP injection in the striatum of 6-hydroxydopamine (6-OHDA)-injured rats. Recently, Moloney et al. designed a fibrin-based scaffold for intracerebral delivery of GDNF overexpressing stem cells [40]. Thrombin and fibrinogen injection resulted in the in situ formation of fibrin in rat brains. Fibrin provided a substrate for the stem cells that were capable of secreting the neurotrophic factor. Fibrin biomaterial showed neither adverse effects on cell survival nor immunoreaction. Further studies are necessary to confirm the potential of this scaffold. In another study, Requejo et al. assessed the topographical distribution of morphological changes in the rat brain after the local injection of nanoencapsulated neurotrophic factors [41]. Synergistic combination of neurotrophic factors provided neuronal preservation and nigrostriatal axon restoration while proliferation assays confirmed the presence of immature cells in the subventricular zone. 


\subsection{DDS for systemic delivery}

Systemic delivery of certain DDS has the potential to cross the BBB and target specific brain regions. Coating DDS with surfactants such as polysorbate 80, dextran or polyethylene glycol (PEG) has been recently reviewed concerning neurodegenerative disorder applications [42]. Taking advantage of BBB endogenous receptors appears to be a suitable strategy to cross the BBB. With this aim, NPs were conjugated with transferrin, insulin, glutathione, LDL, angioPEP, hydroxibutiric acid or antibodies, and successful delivery of drugs across the BBB was achieved (revised in [43]). Currently, there is an emerging interest in the development of non-invasive methods to bypass the $\mathrm{BBB}$ and recent efforts have concentrated on the intranasal administration of DDS to the brain.

\section{$\underline{D D S \text { for dopamine agonist, protein inhibitor and peptide delivery }}$}

A large number of studies have been performed using DDS to obtain sustained levels of active DA in the brain. For instance, Pahuja et al. designed DA-loaded poly(lacticcoglycolic acid) (PLGA) NPs for intravenous delivery [44]. DA-PLGA-NPs crossed the $\mathrm{BBB}$, reached the striatum and reversed functional deficits in 6-OHDA-lesioned rats. The constant slow release of DA allows the reduction of quinone adduct formation, DA autooxidation and other toxic events when compared to bulk DA administration. Gambaryan et al. developed PLGA-NPs for intranasal administration of L-DOPA [45]. In vivo studies confirmed a long-lasting effect of L-DOPA restoring the motor impairments compared to the free-drug. Di Gioia et al. developed intranasal DA delivery using glycol chitosan/sulfobytylether-b-cyclodextrin based NPs [46]. Ethylene glycol confers hydrophilic properties whereas chitosan-thiomer derivates give mucoadhesive attributes. The presence of the anionic sulfobytylether-b-cyclodextrin such as crosslinking agent was found to have a potential ability to stabilize DA. Mucociliary clearance turns out to be the most usual added difficulty when dealing with this type of administration. In order to reduce this inconvenience, substance P-loaded NPs modified with gelatin were proposed [47]. Gelatin confers a negative charge that may prolong NPs' residence time in the mucociliar tract. In vivo studies also confirmed the neuroprotective role of substance $\mathrm{P}$ against 6-OHDA-induced apoptosis. Another nose to brain study that demonstrates enhanced brain targeting using DDS is that of Mittal et al., who prepared rasagiline-loaded chitosan glutamate NPs by ionic gelation [48]. Bioavailability assays determined a significant enhancement of this MAO inhibitor in the CNS. Similar results were obtained encapsulating DA agonist ropinirole in mucoadhesive NPs [49]. These studies demonstrated superiority over other delivery routes due to a prolonged and targeted brain effect. However, further in vivo studies should be accomplished to ensure the safety and efficacy of this route for chronic administration in PD patients.

\section{DDS for gene therapy}


Viral gene delivery systems exhibit limitations and disadvantages including mutagenesis, carcinogenesis, and immune response. In order to avoid these issues viruses can be replaced by nanocarriers. In this sense, Liu et al. developed PEGpolyethyleneimine NPs for $\alpha$-syn siRNA delivery [50]. A significant decrease in endogenous $\alpha$-syn was obtained by SNCA gene silencing in vitro. These complexes demonstrated high transfection and cytoprotective effect after inducing MPP+ cell apoptosis. Nevertheless, this promising gene therapy needs to be validated in vivo before definitive conclusions can be drawn. In another study, Huang et al. developed dendrigraft poly-lysine NPs loaded with hGDNF gene [51]. In order to cross the BBB, NPs were conjugated with PEG and AngioPEP, a small peptide able to interact with endogenous $\mathrm{BBB}$ receptors facilitating $\mathrm{BBB}$ crossing. Multiple intravenous administrations improved the recovery of rotenone induced parkinsonian rats. Recently, polyethylene imine grafted chitosan nanocarriers for GDNF gene delivery were assayed [52] demonstrating a high in vitro GDNF gene expression and confirming the biocompatibility of this approach. Therefore this application should be borne in mind to design future in vivo evaluations.

\section{$\underline{D D S}$ for neuroprotector and antioxidant delivery}

The lack of the natural antioxidants catalase, glutathione and superoxide dismutase, among others, in midbrain regions of parkinsonian patients is thought to be linked to PD pathogenesis. Thus, antioxidant approaches are being investigated as potential strategies to detain oxidative stress triggered by ROS. For instance, Da Rocha Lindner et al. reported an improved neuroprotective effect of resveratrol-loaded PLA NPs coated with polysorbate 80 compared with the free drug in vivo [53]. Haney et al. developed catalase loaded exosomes [54]. These non-toxic nanosized vesicles (100-200 nm) with immune privilege status are secreted by cells for long distance intercellular communications. Tetraspanins and integrins on exosomes surface yield BBB crossing and internalization via endocytosis. In vivo studies demonstrate a reduction of brain inflammation after intranasal and intravenous administration. This innovative approach deserves further investigation with a view to clinical CNS delivery.

Esteves et al. demonstrated the neuroprotective effect of nanoencapsulated retinoic acid on a MPTP-mouse model of PD. Polymeric NPs were prepared by electrostatic interaction of polyethylenimine complexed with retinoic acid and dextran sulfate [55]. Retinoic acid NPs may induce the expression of mRNA and the protein transcription factors Nurr1 and Pitx3, responsible for DA neuronal survival and development. However, retinoic acid controlled release must be assayed and clarified sufficiently, for obvious safety reasons.

\section{DDS for neurotrophic factor delivery}


A new nose to brain non-invasive delivery of neurotrophic factor was developed by Zhao et al. combining the advantages of polymeric NPs, fat emulsions, and liposomes [56]. A functional recovery in hemiparkinsonian rats was observed by encapsulating basic fibroblast growth factor in phospholipid-based gelatin NPs. The protein level of this growth factor in different regions of rat brain suggested that exogenous neurotrophic factor could reach the olfactory bulb and striatum via nasal epithelium without damaging the mucous membrane.

\section{CONCLUDING REMARKS}

PD pathogenesis still remains unclear and the molecular basis operating in the pathways of DAergic cell death are unknown. Moreover, aging acts on many cellular processes influencing PD progression. In order to modify the progression of the disease, understanding the exact relationship between age and PD may create opportunities for intervention. Animal models are the best way to understand PD pathogenesis and also to solve some questions related to disease, for example the role of LBs or the vulnerability of certain DAergic neurons to neurodegeneration. However, current animal models do not recapitulate all the features that take place in the human PD brain. Consequently, animal models that resemble the disease features in a more realistic way are required.

In addition, understanding the underlying mechanisms of PD etiology will be the key to identifying molecular targets for innovative treatments. In this sense, targeting $\alpha$-syn for therapeutic intervention has been extensively investigated. However, since the exact role of $\alpha$-syn is not totally understood, care must be taken when decreasing $\alpha$-syn nonspecifically. Perhaps it would be better to up-regulate the intrinsic clearance mechanisms of toxic $\alpha$-syn, like the lysosomal system.

Concerning current PD treatments, most of them are aimed at addressing motor symptoms, but there is no therapy focused on modifying the course of the disease. In addition, certain non-motor symptoms with a great impact on the quality of life in PD patients do not always respond to DAergic drugs. Thus, there is an urgent need to develop both symptomatic and disease-modifying therapies. Most probably, a combination of drugs with different mechanisms of action may be necessary, probably all of them combined within a DDS. As demonstrated in this review, the use of DDS presents enormous benefits for drug administration, and may ultimately result in better therapies for PD. Advances in DDS use have opened up unprecedented possibilities given their ability to enhance drug potential while reducing the side effects. However, additional work is required before any of these promising strategies will become available in clinical practice bringing major benefits to PD patients.

\section{REFERENCES}

[1] Kalia L V, Lang AE. Parkinson's disease. Lancet 2015;6736:1-17. 
[2] Mosharov E V, Larsen KE, Kanter E, Phillips KA, Wilson K, Schmitz Y, et al. Interplay between cytosolic dopamine, calcium, and alpha-synuclein causes selective death of substantia nigra neurons. Neuron 2009;62:218-29.

[3] Yacoubian TA, Standaert DG. Targets for neuroprotection in Parkinson's disease. Biochim Biophys Acta 2009;1792:676-87.

[4] Dehay B, Bourdenx M, Gorry P, Przedborski S, Vila M, Hunot S, et al. Targeting $\alpha$-synuclein for treatment of Parkinson's disease: mechanistic and therapeutic considerations. Lancet Neurol 2015:855-66.

[5] Spillantini MG, Schmidt ML, Lee VM-Y, Trojanowski JQ, Jakes R, Goedert M. [alpha]-Synuclein in Lewy bodies 1997;388:839-40.

[6] Van Den Eeden SK, Tanner CM, Bernstein AL, Fross RD, Leimpeter A, Bloch D a, et al. Incidence of Parkinson's disease: variation by age, gender, and race/ethnicity. Am J Epidemiol 2003;157:1015-22.

[7] Beilina A, Cookson MR. Genes associated with Parkinson's disease: regulation of autophagy and beyond. J Neurochem 2015

[8] Collier T, Kanaan N, Kordower J. Ageing as a primary risk factor for Parkinson's disease: evidence from studies of non-human primates. Nat Rev Neurosci 2011;12:359-66.

[9] Rodriguez M, Morales I, Rodriguez-Sabate C, Sanchez A, Castro R, Brito JM, et al. The degeneration and replacement of dopamine cells in Parkinsonâ€ $€^{\mathrm{TM}_{S}}$ disease: the role of aging. Front Neuroanat 2014;8:1-7.

[10] Yahr MD, Duvoisin RC, Schear MJ, Barrett RE, Hoehn MM. Treatment of parkinsonism with levodopa. Arch Neurol 1969;21:343-54.

[11] Cotzias GC. Levodopa in the treatment of Parkinsonism. JAMA 1971;218:19038 .

[12] Maetzler W, Liepelt I, Berg D. Progression of Parkinson's disease in the clinical phase: potential markers. Lancet Neurol 2009;8:1158-71.

[13] Todorova A, Jenner P, Ray Chaudhuri K. Non-motor Parkinson's: integral to motor Parkinson's, yet often neglected. Pract Neurol 2014:1-13.

[14] Seppi K, Weintraub D, Coelho M, Perez-Lloret S, Fox SH, Katzenschlager R, et al. The Movement Disorder Society Evidence-Based Medicine Review Update: Treatments for the non-motor symptoms of Parkinson's disease. Mov Disord 2011;26 Suppl 3:S42-80.

[15] Garbayo E, Ansorena E, Blanco-Prieto MJ. Drug development in Parkinson's disease: from emerging molecules to innovative drug delivery systems. Maturitas 2013;76:272-8. 
[16] Burré J, Sharma M, Südhof TC. $\alpha$-Synuclein assembles into higher-order multimers upon membrane binding to promote SNARE complex formation. Proc Natl Acad Sci 2014;111:E4274-83.

[17] Looyenga BD, Brundin P. Silencing synuclein at the synapse with PLK2. Proc Natl Acad Sci U S A 2013;110:16293-4.

[18] Inglis KJ, Chereau D, Brigham EF, Chiou SS, Schöbel S, Frigon NL, et al. Pololike kinase 2 (PLK2) phosphorylates $\alpha$-synuclein at serine 129 in central nervous system. J Biol Chem 2009;284:2598-602.

[19] Li J, Ma W, Wang PY, Hurley PJ, Bunz F, Hwang PM. Polo-like kinase 2 activates an antioxidant pathway to promote the survival of cells with mitochondrial dysfunction. Free Radic Biol Med 2014;73:270-7.

[20] Cizmecioglu O, Warnke S, Arnold M, Duensing S, Hoffmann I. Plk2 regulated centriole duplication is dependent on its localization to the centrioles and a functional polo-box domain. Cell Cycle 2008;7:3548-55.

[21] Zhang Q, Kim Y-C, Narayanan NS. Disease-modifying therapeutic directions for Lewy-Body dementias. Front Neurosci 2015;9:1-9.

[22] Brundin P, Atkin G, Lamberts JT. Basic science breaks through: New therapeutic advances in Parkinson's disease. Mov Disord 2015;30:1521-7.

[23] Zhou C, Przedborski S. Intrabody and Parkinson's disease. Biochim Biophys Acta - Mol Basis Dis 2009;1792:634-42.

[24] Deger JM, Gerson JE, Kayed R. The interrelationship of proteasome impairment and oligomeric intermediates in neurodegeneration. Aging Cell 2015;14:715-24.

[25] Pan T, Kondo S, Le W, Jankovic J. The role of autophagy-lysosome pathway in neurodegeneration associated with Parkinson's disease. Brain 2008;131:1969-78.

[26] Xilouri M, Brekk OR, Kirik D, Stefanis L. LAMP2A as a therapeutic target in Parkinson disease. Autophagy 2013;9:2166-8.

[27] Tofaris GK. Lysosome-dependent pathways as a unifying theme in Parkinson's disease. Mov Disord 2012;27:1364-9.

[28] Dehay B, Martinez-Vicente M, Caldwell G a., Caldwell K a., Yue Z, Cookson MR, et al. Lysosomal impairment in Parkinson's disease. Mov Disord 2013;28:725-32.

[29] Murphy KE, Gysbers AM, Abbott SK, Spiro AS, Furuta A, Cooper A, et al. Lysosomal-associated membrane protein 2 isoforms are differentially affected in early Parkinson's disease. Mov Disord 2015;30:1639-47. doi:10.1002/mds.26141. 
[30] Lee BD, Shin J-H, VanKampen J, Petrucelli L, West AB, Ko HS, et al. Inhibitors of leucine-rich repeat kinase-2 protect against models of Parkinson's disease. Nat Med 2010;16:998-1000.

[31] Hang L, Thundyil J, Lim K-L. Mitochondrial dysfunction and Parkinson disease: a Parkin-AMPK alliance in neuroprotection. Ann N Y Acad Sci 2015;1350:3747.

[32] Bir A, Sen O, Anand S, Khemka VK, Banerjee P, Cappai R, et al. $\alpha$-synucleininduced mitochondrial dysfunction in isolated preparation and intact cells: Implications in the pathogenesis of Parkinson's disease. J Neurochem 2014;131:868-77.

[33] Chan CS, Gertler TS, Surmeier DJ. Calcium homeostasis, selective vulnerability and Parkinson's disease. Trends Neurosci 2009;32:249-56.

[34] Umemura A, Oeda T, Yamamoto K, Tomita S, Kohsaka M. Baseline Plasma CReactive Protein Concentrations and Motor Prognosis in Parkinson Disease 2015;41:1-12.

[35] Kang KS, Lee S-I, Hong JM, Lee JW, Cho HY, Son JH, et al. Hybrid scaffold composed of hydrogel/3D-framework and its application as a dopamine delivery system. J Control Release 2014;175:10-6.

[36] Garbayo E, Montero-Menei CN, Ansorena E, Lanciego JL, Aymerich MS, Blanco-Prieto MJ. Effective GDNF brain delivery using microspheres-A promising strategy for Parkinson's disease. J Control Release 2009;135:119-26.

[37] Garbayo E, Ansorena E, Lanciego JL, Blanco-Prieto MJ, Aymerich MS. Longterm neuroprotection and neurorestoration by glial cell-derived neurotrophic factor microspheres for the treatment of Parkinson's disease. Mov Disord 2011;26:1943-7.

[38] Garbayo E, Lana H, D’Addario G, Carmona-Abellan MM, Marcilla I, Lanciego JL, et al. Parkinsonian monkeys showed brain structural and functional recovery following local delivery of microencapsulated GDNF. Present. Annu. Meet. Control. Release Soc., Edinburgh: 2015.

[39] Herrán E, Requejo C, Ruiz-Ortega JA, Aristieta A, Igartua M, Bengoetxea H, et al. Increased antiparkinson efficacy of the combined administration of VEGFand GDNF-loaded nanospheres in a partial lesion model of Parkinson's disease. Int J Nanomedicine 2014;9:2677-87.

[40] Moloney TC, Ní Fhlathartaigh M, Kulkarni M, Pandit A, Dowd E. Fibrin As a Scaffold for Delivery of GDNF Overexpressing Stem Cells to the Adult Rat Brain. ACS Biomater Sci Eng 2015;1:559-66.

[41] Requejo C, Ruiz-Ortega JA, Bengoetxea H, Garcia-Blanco A, Herrán E, Aristieta A, et al. Topographical Distribution of Morphological Changes in a Partial Model 
of Parkinson's Disease-Effects of Nanoencapsulated Neurotrophic Factors Administration. Mol Neurobiol 2015;52:846-58.

[42] Singh D, Kapahi H, Rashid M, Prakash A, Majeed ABA, Mishra N. Recent prospective of surface engineered Nanoparticles in the management of Neurodegenerative disorders. Artif Cells, Nanomedicine, Biotechnol 2015:1-12.

[43] Leyva-Gómez G, Cortés H, Magaña JJ, Leyva-García N, Quintanar-Guerrero D, Florán B. Nanoparticle technology for treatment of Parkinson's disease: the role of surface phenomena in reaching the brain. Drug Discov Today 2015;20.

[44] Pahuja R, Seth K, Shukla A, Shukla RK, Bhatnagar P. Trans-blood brain barrier delivery of dopamine-loaded nanoparticles reverses functional deficits in parkinsonian rats. ACS Nano 2015;9:4850-71.

[45] Gambaryan PY, Kondrasheva IG, Severin ES, Guseva AA, Kamensky AA. Increasing the Efficiency of Parkinson's Disease Treatment Using a poly(lacticco-glycolic acid) (PLGA) Based L-DOPA Delivery System. Exp Neurobiol 2014;23:246-52.

[46] Di Gioia S, Trapani A, Mandracchia D, De Giglio E, Cometa S, Mangini V, et al. Intranasal delivery of dopamine to the striatum using glycol chitosan/sulfobutylether- $\beta$-cyclodextrin based nanoparticles. Eur J Pharm Biopharm 2015;94:180-93.

[47] Lu C-T, Jin R, Jiang Y, Lin Q, Yu W-Z, Mao K-L, et al. Gelatin nanoparticlemediated intranasal delivery of substance $P$ protects against 6-hydroxydopamineinduced apoptosis: an in vitro and in vivo study. Drug Des Devel Ther 2015;9:1955-62.

[48] Mittal D, Md S, Hasan Q, Fazil M, Ali A, Baboota S, et al. Brain targeted nanoparticulate drug delivery system of rasagiline via intranasal route. Drug Deliv 2015:1-10.

[49] Jafarieh O, Md S, Ali M, Baboota S, Sahni JK, Kumari B, et al. Design, characterization, and evaluation of intranasal delivery of ropinirole-loaded mucoadhesive nanoparticles for brain targeting. Drug Dev Ind Pharm 2014;41:18 .

[50] Liu YY, Yang XY, Li Z, Liu ZL, Cheng D, Wang Y, et al. Characterization of polyethylene glycol-polyethyleneimine as a vector for alpha-synuclein siRNA delivery to PC12 cells for Parkinson's disease. CNS Neurosci Ther 2014;20:7685 .

[51] Huang R, Ma H, Guo Y, Liu S, Kuang Y, Shao K, et al. Angiopep-conjugated nanoparticles for targeted long-term gene therapy of parkinson's disease. Pharm Res 2013;30:2549-59.

[52] Lai P, Wu H, Tseng T. Glial cell line-derived neurotrophic factor gene delivery via a polyethylene imine grafted chitosan carrier 2014:3163-74. 
[53] Da Rocha Lindner G, Bonfanti Santos D, Colle D, Gasnhar Moreira EL, Daniel Prediger R, Farina M, et al. Improved neuroprotective effects of resveratrolloaded polysorbate 80-coated poly(lactide) nanoparticles in MPTP-induced Parkinsonism. Nanomedicine (Lond) 2015;10:1127-38.

[54] Haney MJ, Klyachko NL, Zhao Y, Gupta R, Plotnikova EG, He Z, et al. Exosomes as drug delivery vehicles for Parkinson's disease therapy. J Control Release 2015;207:18-30.

[55] Esteves M, Cristóvão AC, Saraiva T, Rocha SM, Baltazar G, Ferreira L, et al. Retinoic acid-loaded polymeric nanoparticles induce neuroprotection in a mouse model for Parkinson's disease. Front Aging Neurosci 2015;7:1-10.

[56] Zhao YZ, Li X, Lu CT, Lin M, Chen LJ, Xiang Q, et al. Gelatin nanostructured lipid carriers-mediated intranasal delivery of basic fibroblast growth factor enhances functional recovery in hemiparkinsonian rats. Nanomedicine Nanotechnology, Biol Med 2014;10:755-64. 


\section{Contributors}

CRN, EG, and MMCA did the scientific literature review and wrote the first draft of the article. CRN and EG created the figures. RML critically revised the manuscript. MJBP edited the paper and provided the final version. All authors read and approved the final manuscript.

\section{Competing interest}

We declare no financial conflicts of interest.

\section{Funding}

The authors have received no funding for this article 


\section{FIGURE LEGENDS}

Figure 1: New therapeutic targets for PD treatment including emerging pharmacological disease modifying strategies to detain dopamine neuron death (green). (A) Alpha-synuclein ( $\alpha$-syn) aggregation and phosphorylation. $\alpha$-syn natural monomer or tetramer conformation and pathologic aggregation equilibrium. Passive or active immunization or phosphorylation inhibition may detain oligomerization and fibrillation as well as transcellular propagation. (B) Proteosome lysosome approach. $\alpha$ syn proteasome and lysosome defective cleareance. $\alpha$-syn is a substrate of cytosolic chaperone protein that binds LAMP2A allowing chaperone mediated autophagy. $\alpha$-syn is ubiquitinated by E3 ubiquitin protein ligase for proteosome recognition and degradation. (C) Mitochondrial disruptions and oxidative stress. Defective axonal transport or fussion/fission processes, mitophagy impairment and $\alpha$-syn mitochondrial membrane interaction among others may lead to mitochondrial disruptions. Calcium dyshomeostasis between endoplasmic reticule and mitochondria or free radical accumulation triggers neuronal degeneration. (D) Neuroinflammation and glial environment. Microglia secrete cytokines generating a neuroinflammatory environment. Astrocyte release chemokines to regulate glial environment and secrete antioxidants as well as neurotrophic factors to promote gliogenesis and neuronal reparation.

Figure 2: A) Surface-modified NP. Drug is embedded in a degradable or nondegradable matrix allowing controlled release and drug protection. (B) Image of transmission electronic microscope (TEM) showing polymeric nanoparticles under investigation for PD treatment. 


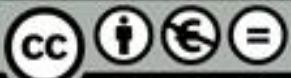

BY NC ND Esta obra está bajo una licencia de Creative Commons Reconocimiento-

NoComercial-SinObraDerivada 4.0 Internacional. 